\title{
Direct Observation of Localized Defect States in Semiconductor Nanotube Junctions
}

\author{
Hajin Kim, ${ }^{1}$ J. Lee, ${ }^{1}$ S.-J. Kahng, ${ }^{2}$ Y.-W. Son, ${ }^{1}$ S. B. Lee, ${ }^{1}$ C.-K. Lee, ${ }^{1}$ J. Ihm, ${ }^{1}$ and Young Kuk ${ }^{1, *}$ \\ ${ }^{1}$ School of Physics and Center for Science in Nanometer Scale, Seoul National University, Seoul 151-742, Korea \\ ${ }^{2}$ Department of Physics, Korea University, Seoul, 136-701, Korea.
}

(Received 14 February 2003; published 29 May 2003)

\begin{abstract}
We report scanning tunneling microscopy of semiconductor-semiconductor carbon nanotube junctions with different band gaps. Characteristic features of the wave functions at different energy levels, such as a localized defect state, are clearly exhibited in the atomically resolved scanning tunneling spectroscopy. The peaks of the Van Hove singularity on each side penetrate and decay into the opposite side across the junction over a distance of $\sim 2 \mathrm{~nm}$. These experimental features are accounted for, with the help of tight-binding calculation, by the presence of pentagon-heptagon pair defects at the junction.
\end{abstract}

As the critical lengths of active electronic devices shrink down to nanometer scales, we face several limiting problems such as accurate patterning and processing of the parts, routing of the interconnection wires, and the clock frequency. It has been suggested that onedimensional (1D) devices made of functionalized 1D wires such as carbon nanotubes (CNTs) may resolve these problems [1,2] ever since their discovery [3]. Spatially functionalized CNTs can now be fabricated utilizing defects [4,5], adsorbed or inserted molecules [6], or chemical doping $[7,8]$. While metal-semiconductor (MS) CNT junctions and selectively doped semiconducting CNTs have been proposed to be used for active electrical devices $[4,5,9,10]$, semiconductor-semiconductor (SS) CNT junctions with different band gaps can be used to produce quantum electro-optical devices. In the present work, we are interested in intramolecular SS CNT junctions with native defects (pentagons and heptagons) connecting smoothly two CNTs of different diameters. The sizes of band gaps and the gradual shaping of chemical potentials around the junctions are crucial in designing active devices. To study these properties, we have probed the "atomically resolved" electronic structure of SS CNT junctions experimentally with scanning tunneling spectroscopy (STS) supported by theoretical simulations based on tight-binding calculation.

Single-wall CNTs made with the laser ablation method were sonicated for $>1 \mathrm{~h}$ in dichloroethane to produce a colloidal suspension after settling down, which remains intact for months. A droplet of this suspension was dispersed on the $A u(111)$ surface precleaned by repeated cycles of sputtering and annealing in an ultrahigh vacuum (UHV) chamber. To avoid contamination in an ambient environment, we performed this procedure in a glovebox filled with pure Ar gas connected to the UHV chamber through a load lock. In the UHV chamber, the sample was heated again up to $\sim 150^{\circ} \mathrm{C}$ to remove residual solvent. When we probed the sample with a home-built low temperature scanning tunneling microscope [11] which can be operated at 5 or $80 \mathrm{~K}$, isolated and bundled
CNTs on the $\mathrm{Au}(111)$ terrace were readily observed as in Fig. 1(a). Occasionally, intramolecular junctions with native defects could be distinguished from others due to their physical bending or characteristic features in atomically resolved scanning tunneling microscopy (STM) images [12,13]. It is known that an intramolecular junction can be grown by fusing two neighboring CNTs with a high electric field [14], but we performed the present experiment on naturally formed junctions. The types of the junctions could be determined by measuring $d I / d V$ spectra on both sides.

Figures 1(b)-1(d) show bias-dependent STM images taken on an SS CNT junction. At negative sample bias voltages $(V)$, the defect is not so conspicuous, but at positive voltages more remarkable modification of the electron density can be observed on the surface. Especially at $V=0.5 \mathrm{~V}$, the pattern of the surface corrugation is very different from other bias voltages; the defective region reveals a pattern of longer period, very similar to the $(\sqrt{3} \times \sqrt{3}) R 30^{\circ}$ structure observed near the defects on graphite surfaces [15]. As we will discuss in the STS results, this pattern is caused by additional defect states. The presence of such localized defect states

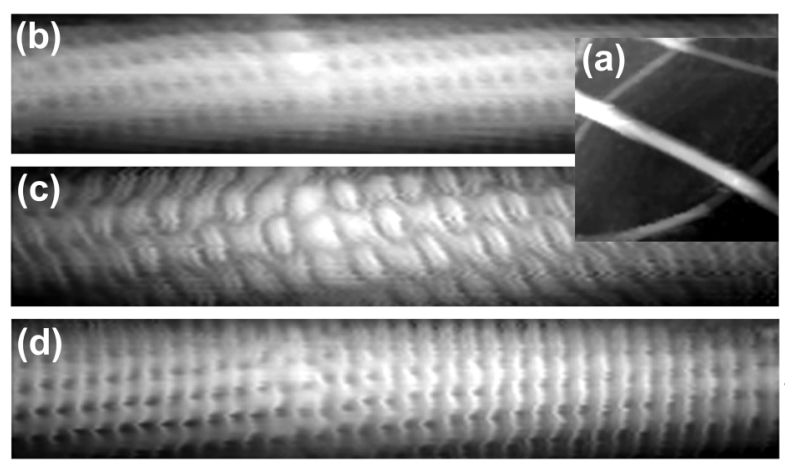

FIG. 1. (a) A $100 \times 100 \mathrm{~nm}$ STM image. (b),(c),(d) STM images of a carbon nanotube intramolecular junction at three different sample bias voltages, (b) $1.5 \mathrm{~V}$, (c) $0.5 \mathrm{~V}$, and (d) $-0.3 \mathrm{~V}$. The length of these images is $7.6 \mathrm{~nm}$. 
has been predicted $[9,16]$ and observed for metal-metal CNT junctions [5], but not yet for SS CNT junctions.

Since the tunneling current $(I)$ of an STM reflects the integrated local density of states (LDOS) of the sample from the Fermi level to the sample bias voltage $V$, the value of $d I / d V$ is approximately proportional to the LDOS at this energy level [17]. In the $d I / d V$ spectrum obtained above the left-hand side of the junction (Fig. 2), the first and the second peaks of the Van Hove singularity (VHS) in the occupied and the unoccupied states are visible. $[(V / I)(d I / d V)$ is often used to normalize the bias-dependent tunneling probability.] The LDOS of the junction was spatially resolved by taking $d I / d V$ spectra along the CNT at a fixed tunneling gap or a constant tunneling current. In the present experiment, the STM tip was repeatedly scanned over the topmost line on the same nanotube, leaving the reference current of feedback fixed at $0.5 \mathrm{nA}$, and changing the bias voltage by a small increment for each line scan. The map of $V d I / d V$ obtained by lock-in technique with a modulation signal of $\sim 20 \mathrm{mV}$ and $\sim 3 \mathrm{kHz}$ as a function of both the position along the nanotube axis and the sample bias voltage is presented as a two-dimensional image shown in Fig. 2, in which brightness is proportional to the LDOS. The LDOS spectrum at the left end of the imaged region is in good accordance with the point $d I / d V$ spectrum. The band gaps of the left and the right nanotubes were observed as 0.92 and $0.80 \mathrm{eV}$ from the measured VHS peaks, and their chiral indices were estimated to be $(15,2)$ and $(19,3)$,

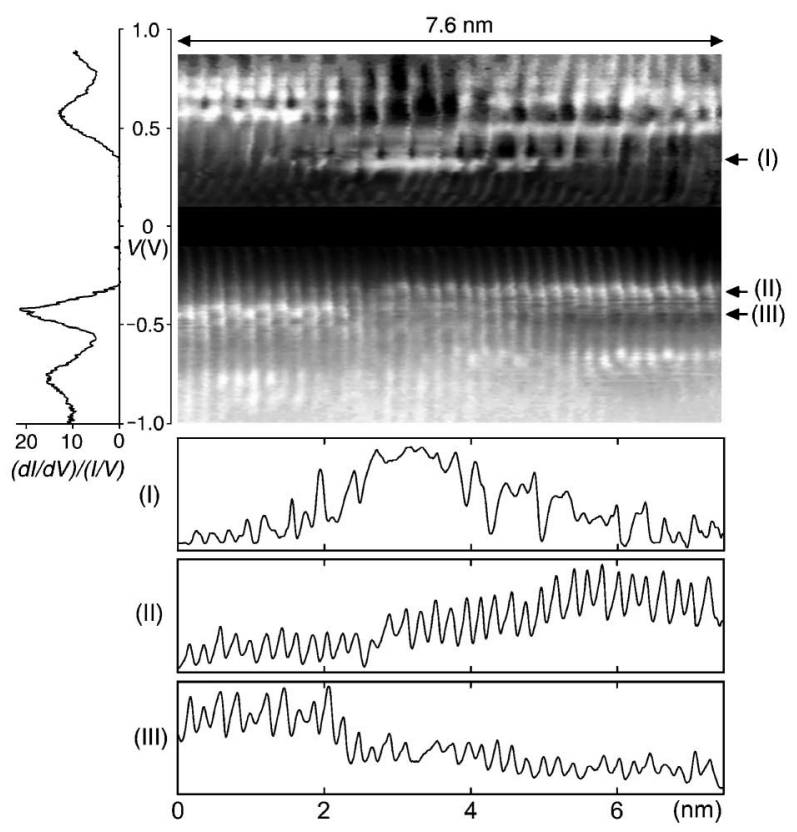

FIG. 2. Spatially resolved LDOS of the junction by STS shown together with point spectroscopy obtained on the left nanotube. The abscissa is the position along the tube axis and the ordinate is the sample bias voltage. Graphs (I), (II), and (III) are the line profiles at the energy levels marked by arrows. respectively, from the corresponding STM images and spectroscopic features. If the band gaps are determined from the edges, they are smaller by $\sim 0.2 \mathrm{eV}$. The measured band gaps are larger than the theoretically expected values for given tube diameters. Such broadening of the energy scale appears quite often when band edges are measured in STS. A periodic vertical pattern reflects the electronic wave functions of CNTs and demonstrates the atomic resolution of the spectroscopy. It also explains the atomic corrugation observed in the topographic images in Fig. 1; especially the elongated periodicity in Fig. 1(c) agrees with that of vertical lines around the defect at positive bias voltages in Fig. 2. For MS CNT junctions created by pentagon-heptagon pair defects, spatially resolved LDOS features were suggested theoretically [9], and observed by point spectroscopy at six spots around one of these junctions [5]. In this junction, the states on both sides decay quickly ( $\sim 2 \mathrm{~nm}$ ) into the other sides without any evidence of defect states in experimental studies.

Two features in this spectroscopic image are worthy of our attention. The first is the localized defect states at $V=$ $0.3 \mathrm{~V}$ which extend over $3 \mathrm{~nm}$, the spatial distribution of which is shown as a line profile labeled as (I) in Fig. 2. This energy level is a little below the conduction band edges of both sides and can be understood, as mentioned previously in Ref. [9], in terms of Hückel's rule that cyclic $\pi$-electron systems with $4 n+2$ ( $n$ : positive integer) $\pi$ electrons are most stable [18]. Six-membered carbon rings are more stable than five- or seven-membered rings, and, thus, a heptagon will try to give up an electron to its neighbors and so plays the role of a donor in a semiconducting nanotube. Similarly, a pentagon works as an acceptor. We were unable to observe obvious localized states below the Fermi level. The second salient feature is the peaks of VHS penetrating and decaying into the opposite side. This is more obvious for the valence band edges at both sides that are less perturbed by the defect state. The spatial distributions of these edge states are shown as line profiles labeled as (II) and (III) in Fig. 2.

To understand these experimental observations, we simulated the topographic STM images and the twodimensional (position and energy) STS map of the SS CNT junction. The electronic structure of the junction was obtained by tight-binding calculation with the $\pi$-orbital interaction energy, $V_{p p \pi}$ of $2.66 \mathrm{eV}$ [19]. A more sophisticated ab initio calculation method could not be employed here because of the huge unit cell size ( 5000 atoms) and many different defect geometries to be tested. The tunneling current between the STM tip and the sample was calculated by a method similar to that of Meunier and Lambin [12]. We basically followed the Tersoff-Hamann theory [20] in which the tip modeled as an $s$-state single atom is coupled with the sample by hopping interaction. To obtain topographic STM images, the tip height at each point was determined recursively to 
make the tunneling current constant. The STS image consisting of $d I / d V$ spectra was calculated by sweeping the bias voltage with the tip position fixed at each point. After an extensive search over various geometric configurations and also other possible combinations of indices satisfying the straight geometry of the junction, we found that the $(15,2)-(19,3)$ junction with two pairs of pentagons and heptagons agrees best with the experiment.

Figure 3(a) shows the proposed atomic model of this $(15,2)-(19,3)$ junction. A complex consisting of one pentagon and two heptagons exists at the concave region, and a pentagon exists at the convex region of the junction. $d I / d V$ spectra were obtained along the solid line and assembled into a two-dimensional image in Fig. 3(b). Its overall characteristics are qualitatively independent of the choice of the scanning line. Figure 3(c) shows the line profiles of selected individual states [labeled as (I), (II), and (III)] along the scanned line, for comparison with experimental data in Fig. 2. The scanning line is on the front side while defects exist on the back side of the tube. The simulated line scan on the back side (not presented here) shows quite different patterns from experiment, suggesting that the scanning was done on the

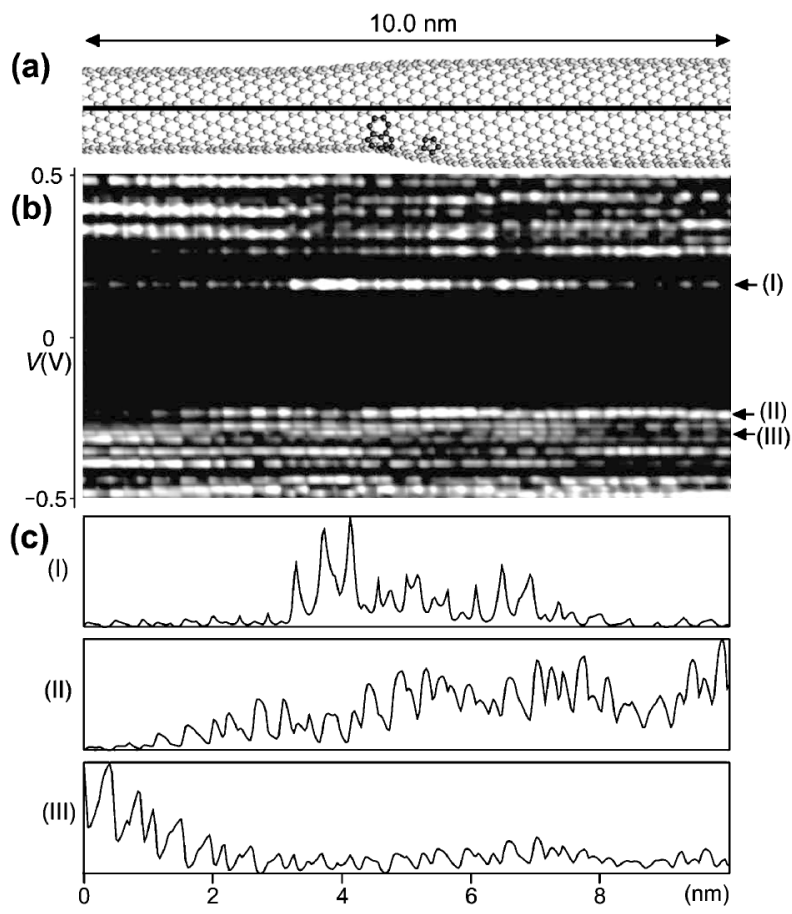

FIG. 3. Simulation of STS on the (15,2)-(19,3) SS CNT junction corresponding to the experimental data in Fig. 2. (a) Atomic model of the junction. The STM tip was scanned over the solid line in the simulation and the defects are on the back side of the tube. (b) Two-dimensional image of the calculated LDOS spectra as a function of the position along the tube axis and the bias voltage. (c) The spatial distributions of the defect state above the Fermi level and the valence band edge states of both sides are marked by arrows. defect-free side in the experiment. A localized defect state labeled as (I) exists $0.11 \mathrm{eV}$ below the conduction band minimum of the right nanotube in reasonable agreement with the state (I) in Fig. 2. As the imaginary part of the wave vector of a defect state below the conduction band minimum is proportional to $\left|E_{c}-\varepsilon_{d}\right|^{1 / 2}$, where $\left|E_{c}-\varepsilon_{d}\right|$ is the difference between the conduction band edge and the defect level, it decays faster to the left than to the right. This asymmetry is also exhibited in the experimental results in Fig. 2. Valence band edge states on both sides labeled as (II) and (III) are also observed to have similar spatial distributions to those in the experimental results. They decay into the opposite side with a decay length of $\sim 2 \mathrm{~nm}$ in qualitative agreement with the experiment.

We also found an acceptorlike defect state very close (within $0.01 \mathrm{eV}$ ) to the valence band edge [state (II)] in this calculation. It resolves the reason why only the donorlike state was observed in the experiment; the acceptorlike state is practically in resonance with the valence band edge state and cannot be resolved in the STS measurement. Another interesting point is that, where the amplitude of the state (I) is maximum [bright spots of the state (I)], the amplitudes of the conduction band states are minima [dark regions above the state (I) in energy]. This is observed in the experiment as well (Fig. 2). It indicates that the donorlike defect state is derived from conduction band states in proximity (both in energy and space) and the local charge is conserved to a good approximation.

Simulated STM images for the $(15,2)-(19,3)$ junction for a few bias voltages are shown with the atomic configuration in Fig. 4. For a sufficiently high bias voltage $(\geq 1 \mathrm{~V}$ ), the image reveals a rather uniform hexagonal carbon network and the topological defects are not particularly noticeable. For lower positive bias voltages, however, we observe obvious modification (increase in brightness) around the defective region as well as quite different image patterns, and these observations agree qualitatively with the experimental results in Fig. 1, considering the broadening of the energy scale in the experiment. At $-0.25 \mathrm{~V}$, patterns on the left and the right are different and the vertical pattern is more conspicuous on

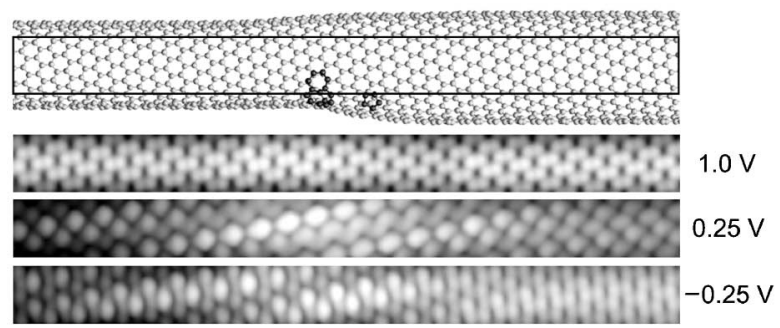

FIG. 4. Calculated topographic images of the (15,2)-(19,3) junction in the solid rectangular region at different sample bias voltages. Defects are located on the back side of the tube. 
the right in agreement with the experimental image at $-0.3 \mathrm{~V}$. These features reflect the difference between the states on the two sides in character and the influence of the defect states.

In summary, we spatially resolved the electronic structure of an SS CNT intramolecular junction by STS and observed a localized defect state decaying into both sides of the junction and the peaks of theVHS decaying into the opposite side of the tube. We interpreted experimental observations in terms of the pentagon-heptagon defects in the junction. The SS CNT junction is a good example of a 1D semiconductor junction. Because of the reduced dimensionality, it is less efficient in screening the electronic states penetrating through the junction than the $3 \mathrm{D}$ materials and has rather large decaying length of the band edge. Once the detailed features of the band gap variation are well understood, we will be able to design single or multiple SS CNT junctions to produce light emitting or laser devices in the future. Equipped with the experimental and theoretical tools used here, one can examine other 1D wires such as boron nitride nanotubes having wider band gap in order to find even better candidate materials for optoelectronic applications.

'This work was supported by the Korean Ministry of Science and Technology through Creative Research Initiatives Program and the Center for Nanotubes and Nanostructured Composites.

*Corresponding author.

Email address: ykuk@phya.snu.ac.kr
[1] C. Dekker, Phys. Today 52, No. 5, 22 (1999).

[2] R. H. Baughman, A. A. Zakhidov, and W. A. de Heer, Science 297, 787 (2002).

[3] S. Iijima, Nature (London) 354, 56 (1991).

[4] Z. Yao, H.W. Ch. Postma, L. Balents, and C. Dekker, Nature (London) 402, 273 (1999).

[5] M. Ouyang, J.-L. Huang, C. . Cheung, and C. M. Lieber, Science 291, 97 (2001).

[6] J. Lee et al., Nature (London) 415, 1005 (2002).

[7] C. Zhou, J. Kong, E. Yenilmez, and H. Dai, Science 290, 1552 (2000).

[8] S. Heinze et al., Phys. Rev. Lett. 89, 106801 (2002).

[9] V. Meunier, P. Senet, and Ph. Lambin, Phys. Rev. B 60, 7792 (1999).

[10] M. S. Ferreira, T. G. Dargam, R. B. Muniz, and A. Latgé, Phys. Rev. B 62, 16040 (2000).

[11] J.-Y. Park, Ph.D. thesis, Seoul National University, 2000.

[12] V. Meunier and Ph. Lambin, Phys. Rev. Lett. 81, 5588 (1998).

[13] D. Orlikowski, M. B. Nardelli, J. Bernholc, and C. Roland, Phys. Rev. B 61, 14194 (2000).

[14] G.W. Ho, A.T. S. Wee, and J. Lin, Appl. Phys. Lett. 79, 260 (2001).

[15] J. Xhie et al., Phys. Rev. B 43, 8917 (1991).

[16] T. Kostyrko, M. Bartkowiak, and G. D. Mahan, Phys. Rev. B 59, 3241 (1999).

[17] J. Tersoff and D. R. Hamann, Phys. Rev. B 31, 805 (1985).

[18] T.W. G. Solomons, Organic Chemistry (Wiley, New York, 1996), 6th ed., p. 624

[19] X. Blase, L. X. Benedict, E. L. Shirley, and S. G. Louie, Phys. Rev. Lett. 72, 1878 (1994).

[20] J. Tersoff and D. R. Hamann, Phys. Rev. Lett. 50, 1998 (1983). 